\title{
Comblike Liquid-Crystalline Polymers from Ionic Complexation of Dendronized Polymers and Lipids
}

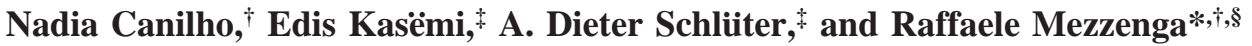 \\ Department of Physics and Fribourg Center for Nanomaterials, University of Fribourg, Pérolles, Ch. \\ du. Musée 3, CH-1700 Fribourg, Switzerland; Department of Materials, Laboratory for Polymer \\ Chemistry, Swiss Federal Institute of Technology, ETH-Zurich, HCI J 541, CH-8093, Zurich, \\ Switzerland; and Nestlé Research Center, Vers-Chez-les-Blancs, 1000, Lausanne 26, Switzerland
}

\begin{abstract}
This work describes the self-assembly behavior, the structure, the state, and phase diagrams of comblike liquid-crystalline polymers obtained by supramolecular ionic complexation of cationic dendronized polymers (PG1-PG3) and anionic sulfonated lipid surfactants. In order to characterize the influence of both the polymer and the surfactant on the microphase separation of these complexes, dendronized polymers with generation $1 \leq n \leq 3$, carrying $2^{n}$ positive charges per monomer, were complexed with a stoichiometric amount of anionic sodium alkyl monosulfate surfactants with hydrocarbon chain of $\mathrm{C}_{8}, \mathrm{C}_{12}, \mathrm{C}_{14}$, and $\mathrm{C}_{18}$ lengths. Supramolecular complexes were obtained in water, and the complexation process was monitored by Fourier transform infrared spectroscopy (FTIR) and nuclear magnetic resonance. After drying and thermal annealing under high vacuum, the complexes showed thermotropic liquid-crystalline behavior as demonstrated by both cross-polarized optical microscopy and differential scanning calorimetry. Small-angle X-ray scattering allowed determining the respective lattice parameters and type of structures for all the complexes considered. Depending on both generation and lipid chain length, amorphous isotropic fluid, columnar rectangular, columnar hexagonal, columnar tetragonal, and lamellar phases were observed, each characterized by a specific order-disorder transition temperature $\left(T_{\mathrm{ODT}}\right)$.
\end{abstract}

\section{Introduction}

Block copolymers have been widely employed during the past 20 years as precursors to template well-ordered periodic nanostructures. ${ }^{1,2}$ The most commonly used type are diblock copolymers. ${ }^{3,4}$ As a consequence of the different chemical nature of the two blocks, these will have a tendency to microphase separate and self-assemble into various nanostructures such as lamellar, cylindrical, double gyroid, and spherical phases. The phase diagrams of these systems have been well established in terms of the composition of the diblock as well as on the segregation parameter, expressed as the product of the FloryHuggins interaction parameter and the polymerization degree of the diblock $(\chi N) .{ }^{5-8}$ In order to explore different possible nanostructures, alternative polymer architectures such as comblike homopolymers or copolymers have been synthesized, ${ }^{9-12}$ for which both the experimental and theoretical phase diagrams have been by far less investigated. In this type of polymer, various liquid-crystalline phases can be induced by changing the type of the mesogenic units attached to the main chain as well as the nature of the polymer backbone. ${ }^{13,14}$ While in this class of comblike polymers the main backbone was essentially linear, other chemical architectures have been studied in recent years. For example, hyperbranched and dendritic macromolecules were used to form the backbone of comblike polymers rather than linear chains. ${ }^{15-20}$

These highly branched macromolecules have a molecular conformation which differs markedly from that of linear polymers, and the flexible or rigid mesogenic units covalently attached to the end-functional groups are selectively distributed

* Corresponding author: e-mail raffaele.mezzenga@unifr.ch, Tel + 41 26300 9066, Fax + 4126300 9747; e-mail raffaele.mezzenga@rdls. nestle.com, Tel + 41217858078 , Fax + 41217858554 .

†niversity of Fribourg, Pérolles.

Swiss Federal Institute of Technology.

$\S$ Nestlé Research Center. on the peripherical external "shell" rather than homogeneously as in the case of linear comblike polymers. Furthermore, the driving forces responsible for the segregation of the mesogenic units and the branched scaffold can be tuned in these systems by changing the generation of the dendrimers or the degree of branching. ${ }^{21}$

Recent efforts in the design of functional comblike-type macromolecules have focused on the synthesis of supramolecules by assembling polymer chains and mesogenic units by hydrogen bonds or ionic complexation rather than covalent bonding. ${ }^{22-25}$ In the hydrogen-bonding strategy use was made mostly of linear homopolymers and blocks copolymers as polymeric building blocks. ${ }^{26}$ In the case of ionic complexation, various polyelectrolyte architectures such as linear and hyperbranched polymers as well as dendrimers have been explored as macromolecular templates and complexed with ionic mesogenes such as hydrocarbon-based surfactants and rigid rods. ${ }^{27-30}$ Biopolyelectrolytic systems have also been studied as comblike polymers templates for complexation with ionic mesogenes in investigations relevant to $\mathrm{pH}$-responsive systems and artificial biomembranes. ${ }^{31}$ Recently, we have reported the use of cationic dendronized polymers as a model system to design comblike liquid-crystalline polymers where microphase-segregated structures can be tailored by both the mesogen type and length as well as the generation of the dendronized polymer. ${ }^{32-34}$ While in these systems the ionically attached mesogene units are expected to play a role similar to that of other comblike supramolecular systems, the generation of the dendronized polymers introduces an additional opportunity to control and tailor the self-assembly behavior of these materials. Indeed, it has been shown that the generation of the dendrons grafted to the backbone of the polymer can be responsible for substantial conformational changes of the chain and systematic increase of the persistence length, driving the polymer from a coiled chain at low generation to a rodlike polymer for high dendron 
generations. ${ }^{35}$ These effects are then expected to be further enhanced by the complexation of the dendrons attached to the polymer backbone with ionic surfactants.

In this work, we discuss the self-assembly, thermotropic behavior, and liquid-crystalline structures of supramolecular complexes formed between cationic polyelectrolytic dendronized polymers ${ }^{36-38}$ and anionic hydrocarbon surfactants of various lengths. Each repeat unit of the dendronized polymer carries a dendron of generation $n$, with $1 \leq n \leq 3$ and with $2^{n}$ positively charged ammonium units. The volume fraction of the surfactant in the complex is controlled by both the dendron generation as well as by the length of the surfactant tail selected. A detailed state and phase diagram of the different liquid-crystalline phases observed at the equilibrium for these complexes are given and discussed in terms of temperature, generation, and alkyl tail length.

\section{Experimental Section}

2.1. Materials. Deionized QMillipore water, ethanol, and 1-butanol analytical grade were used as received from Fluka. Four different anionic sulfated surfactant salts were selected to explore the effect of the hydrocarbon chain length on the complexes. Sodium octyl sulfate (C8) (Fluka, 95\%) represented the shortest hydrocarbon chain considered; sodium dodecyl sulfate (C12, Fluka, 98\%) and sodium tetradecyl sulfate (C14, Fluka, 95\%) represented the intermediate lengths and sodium octadecyl sulfate (C18) (Fluka, $98 \%$ ) the longest one. The critical micelle concentration $(\mathrm{cmc})$ is 8.3, 2, and $134 \mathrm{mM}$ for $\mathrm{C} 12, \mathrm{C} 14$, and $\mathrm{C} 8$, respectively. ${ }^{39}$ The $\mathrm{cmc}$ value for $\mathrm{C} 18$ is not reported in the literature.

2.2. Synthesis of Cationic Dendronized Polymers. The synthesis of water-soluble dendronized polymers used in the present work follows closely the procedure described elsewhere by Kasëmi et al. ${ }^{38}$ for similar systems, and details on first- and secondgeneration dendronized polymers synthesis have been given in a previous paper. ${ }^{34}$ The synthetic procedures for the third-generation dendronized polymer PG3 and all data regarding its chemical characterization are available in the Supporting Information. The respective molecular weights of these three dendronized polymers are $1550800 \mathrm{~g} / \mathrm{mol}$ for PG1, $2346900 \mathrm{~g} / \mathrm{mol}$ for PG2, and 333000 $\mathrm{g} / \mathrm{mol}$ for PG3.

2.3. Methods. Complexation and Purification Procedures. First-, second-, and third-generation cationic dendronized polymers were selected to form ionic supramolecular complexes, as illustrated in Scheme 1. The glass transition temperature $\left(T_{\mathrm{g}}\right)$ of these dendronized polymers was measured at 59,58 , and $82{ }^{\circ} \mathrm{C}$ for PG1, PG2, and PG3 polymers, respectively. The polymers PG1-PG3 carry 2,4 , and 8 , respectively, positively charged ammonium groups per repeat unit when dissolved in acidic water $(\mathrm{pH}=3-4$ adjusted with $\mathrm{HCl}$ ). The entire set of complexations between sodium alkyl monosulfate surfactants and dendronized polymers was carried out at stoichiometric ratio of positive ammonium and negative sulfate charges. Between 50 and $100 \mathrm{mg}$ of lyophilized PG1 to PG3 was dissolved in $50 \mathrm{~mL}$ of water under continuous stirring. For complexation with $\mathrm{C} 8, \mathrm{C} 12$, and $\mathrm{C} 14$, the equivalent molar mass of salt surfactant needed to maintain stoichiometric conditions was dispersed in water. In order to improve the dispersion of C14, the solution was warmed to $50{ }^{\circ} \mathrm{C}$ for $1 \mathrm{~h}$ under stirring. In all three cases, the water volume was adjusted in order to maintain the surfactant concentrations below the cmc. For the complexation with C18, owing to its low solubility in water, a mixture of 1-butanol/ water/ethanol (87.7 wt \%/10.9 wt \%/1.33 wt \%) was used as a solvent for $\mathrm{C} 18$. The $\mathrm{pH}$ values of both the PG1-PG3 and the surfactant solutions were adjusted with $2 \mathrm{~N} \mathrm{HCl}$ to $\mathrm{pH}=3-4$ in order to maintain all amines positively and the sulfates negatively charged. The respective PG1-PG3 solution was then added dropwise to the surfactant solution under continuous stirring. Progressively, the surfactant solution became turbid, indicating the formation of complexes. ${ }^{40}$ They were collected after removal of water from the centrifugated complex-water dispersion. In order to remove possible excess of unbound surfactant in the PG1-C8 to PG3-C8, PG1-C12 to PG3-C12, and PG1-C14 to PG3-C14, the collected precipitates were successively dissolved in 1-butanol and added dropwise to large excess of acidic water $(\mathrm{pH}=3-4)$ in order to avoid 1-butanol emulsification in water. Iterating the procedure consisting of dissolving the precipitated complex in 1-butanol and reprecipitating it in acidic water allows washing at each step eventual excess of unbound lipid, yielding a pure dendronized polymer-lipid complex. The second precipitate is normally taken as final purified complex. PG1-C18 to PG3-C18 complexes were characterized without further purification, owing to their poor solubility in organic solvents. In order to remove residual water in the final complex, powdered samples were dried under vacuum at room temperature for 3 days.

Fourier Transform Infrared Spectroscopy. Infrared spectra have been recorded with a Bomem Hartmann and Braun MB 155 FTIR spectrometer in attenuated total reflection (ATR) with a ZnSe crystal in a spectral region of $600-4000 \mathrm{~cm}^{-1}$ with a resolution of $2 \mathrm{~cm}^{-1}$. In order to achieve good contact with the cell, the powder of the PG $\boldsymbol{x}$-lipid complex was compressed at room temperature to form a platelet and placed directly in contact with the crystal.

Nuclear Magnetic Resonance (NMR). ${ }^{1} \mathrm{H}$ and ${ }^{13} \mathrm{C}$ NMR spectra were recorded on Bruker AM $300\left({ }^{1} \mathrm{H}: 300 \mathrm{MHz} ;{ }^{13} \mathrm{C}\right.$ : $\left.75 \mathrm{MHz}\right)$ and AV $500\left({ }^{1} \mathrm{H}: 500 \mathrm{MHz} ;{ }^{13} \mathrm{C}: 125 \mathrm{MHz}\right)$ spectrometers at room temperature using chloroform- $d$ as a solvent. ${ }^{19} \mathrm{~F}$ NMR spectra of complexes were done on Bruker AB $250\left({ }^{19} \mathrm{~F}\right.$ : $\left.188 \mathrm{MHz}\right)$ using DMSO- $d_{6}$ and $0.4 \mathrm{nmol}$ of hexafluorobenzene $\left(\mathrm{C}_{6} \mathrm{~F}_{6}\right)$ as internal standard. Relaxation time of $30 \mathrm{~s}$ was selected in order to have good signal-to-noise ratio.

Elemental Analysis. Elemental analyses were performed on a Leco CHN-900 and Leco CHNS-932 instrument using acetanilide/ cholesterol, atropin, and caffeine as reference for calibration. For $\mathrm{Cl}$ determination $5 \mathrm{mM}$ of $\mathrm{AgClO}_{4}$ was used as a reagent. The samples were dried rigorously under vacuum prior to analysis to remove strongly adhering solvent molecules.

Annealing Process. In order to attain the thermodynamic equilibrium, the complexes (with the exception of PG1-C12) were annealed for 3 days in a high-vacuum column $\left(10^{-8} \mathrm{mbar}\right)$ at a temperature larger than the glass transition temperature of the dendronized polymers and the melting temperature of the lipids but lower than the temperature of order/disorder transition $\left(T_{\mathrm{ODT}}\right)$ of the liquid-crystalline structure studied. Typical annealing temperatures ranged between 80 and $150^{\circ} \mathrm{C}$. The PG1-C12 complex could not be properly annealed because the $T_{\mathrm{ODT}}$ was very close to the glass transition temperature of PG1. The $T_{\mathrm{ODT}}$ was measured by temperature-dependent SAXS in separate experiments. Heating the complexes above the $T_{\mathrm{ODT}}$ and recovering the original structures upon cooling below the $T_{\mathrm{ODT}}$ proved the observed structures to by typical of thermodynamic equilibrium.

Cross-Polarized Optical Microscopy (CPOM). Birefringence of the complexes was investigated using a Leica DMLB microscope with polarizing filters equipped with a JVC digital TK-C138050 color video camera. The films were cast on a microscope glass plate from a $4 \mathrm{wt} \%$ solution of complex dissolved in 1-butanol or chloroform. The films were predried over night before being annealed for 3 days at $10^{-8}$ mbar according to the procedure described above. In order to study birefringence as a function of temperature, the annealed film was finally inserted into the quartz glass cell of a Linkam hot stage regulated by a Linkam CSS450 temperature controller.

Differential Scanning Calorimetry (DSC). Thermal analysis measurements were performed using a Mettler Toledo DSC821 differential scanning calorimeter (DSC). Samples of a total weight ranging between 3 and $5 \mathrm{mg}$ were closed into aluminum pans of $40 \mu \mathrm{L}$, covered by a holed cap, and analyzed under a nitrogen atmosphere. The DSC measurements were recorded in a temperature range of $25-160{ }^{\circ} \mathrm{C}$ with a heating rate of $10{ }^{\circ} \mathrm{C} / \mathrm{min}$. Since measurements were performed on samples previously annealed and at thermodynamic equilibrium, the first scan was sufficient to obtain information on first- and second-order thermodynamic transitions, 

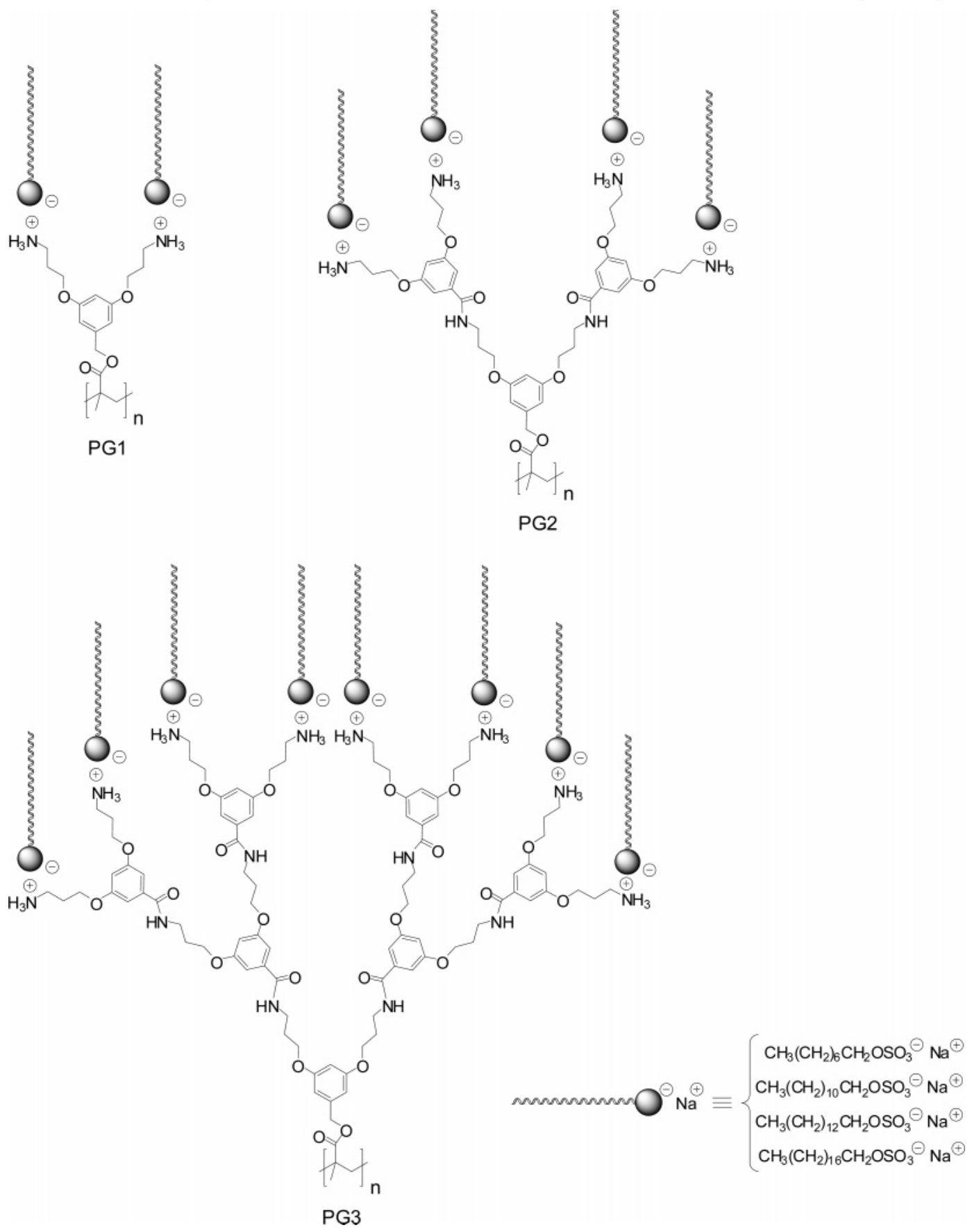

and no second scan curves were run. Different heating rates of DSC scans yielded the same results obtained at $10{ }^{\circ} \mathrm{C} / \mathrm{min}$.

Wide- and Small-Angle X-ray Scattering. Simultaneous wideand small-angle X-rays scattering (SWAXS) experiments were performed using a SAXSess instrument (Anton Paar) with a line collimation setup. The system used a $\mathrm{Cu} K \alpha$ radiation source in a sealed tube $(\lambda=0.1542 \mathrm{~nm})$. The beam was attenuated by a semitransparent nickel foil beam stop. A highly sensitive SWAXS imaging plate slide at $263.3 \mathrm{~mm}$ from the sample is used to collect the signal under vacuum. The sample holder was temperaturecontrolled in the range comprised between 10 and $200{ }^{\circ} \mathrm{C}$. Powder samples were placed in between two mica foils clamped and sandwiched in a steel sample holder for solids samples. Diffraction data were acquired for $1 \mathrm{~h}$ exposure. For temperature-dependent measurements, each temperature was maintained $1 \mathrm{~h}$ prior to data acquisition in order to achieve thermodynamic equilibrium structures. Diffractograms of mica were acquired by SWAXS in order to establish the backgrounds and then subtracted from the sample diffractograms, yielding the data reported in the present paper. The high reproducibility of SAXS measurement was demonstrated by using a set of three different samples per each complex, which resulted in undistinguishable measurements. All the scattering signals were treated with SAXSquant software by Anton Paar.

\section{Results and Discussion}

3.1. Analytical Characterization of the Complexes. The surfactants, the dendronized polymers, and their supramolecular complexes were characterized individually by Fourier transform infrared spectroscopy (FTIR). Figure 1 reports, as an example, the spectra for C12, PG2, and their complex PG2-C12. The absorption band at $1594 \mathrm{~cm}^{-1}$, present in the polymer and absent in the surfactant spectra, is characteristic of protonated ammonium groups. For each ionic complex studied, this band is still present, while the sulfate vibration peaks at 1202 and 1237 $\mathrm{cm}^{-1}$ (both visible in the surfactant spectra) appeared. Although 


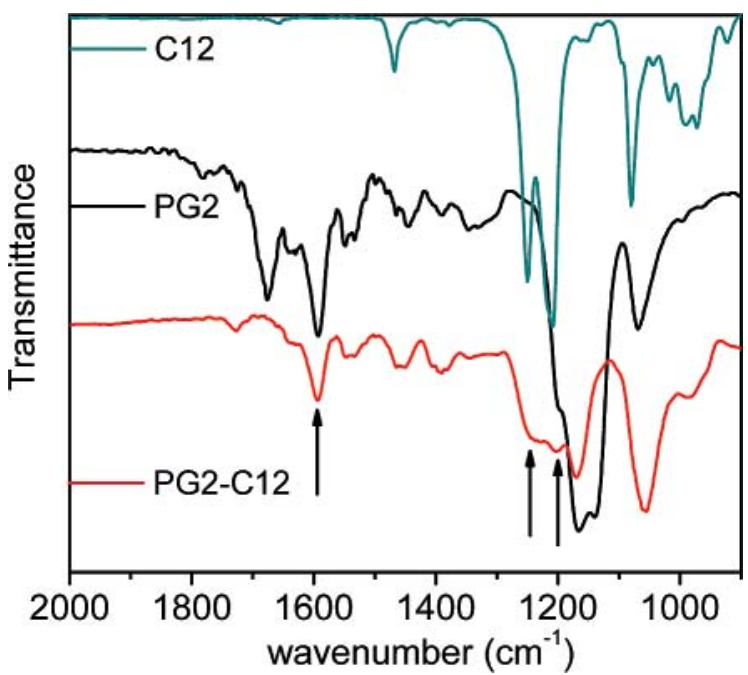

Figure 1. FTIR spectra for a $\mathrm{C} 12$ surfactant alone, PG2 dendronized polymer alone, and the respective PG2-C12 complex. The presence in the complex signals of bands at 1202 and $1237 \mathrm{~cm}^{-1}$ is characteristic of sulfate groups probing the complexation between the PG2 and sulfate surfactants.

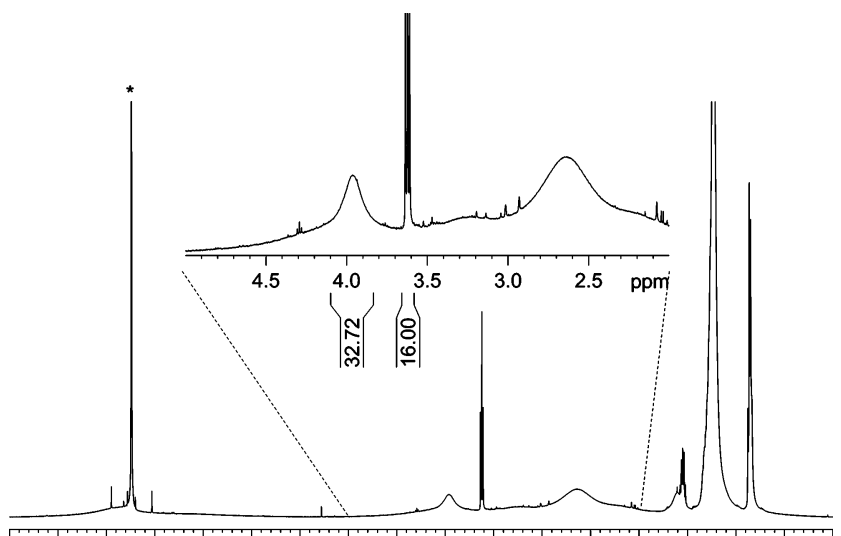

$\begin{array}{llllllllllllllll}8.0 & 7.5 & 7.0 & 6.5 & 6.0 & 5.5 & 5.0 & 4.5 & 4.0 & 3.5 & 3.0 & 2.5 & 2.0 & 1.5 & 1.0 & \mathrm{ppm}\end{array}$

Figure 2. ${ }^{1} \mathrm{H}$ NMR spectrum of PG3-C12 in $\mathrm{CDCl}_{3}$ at room temperature with the expanded range from $2.0<\delta<5.0 \mathrm{ppm}$. The intensities of the $\alpha-\mathrm{CH}_{2}$ signals of the surfactant at $\delta=3.65$ and the one due to all $\mathrm{CH}_{2} \mathrm{O}$ groups of $\mathbf{P G 3}$ at $\delta=3.95$ are compared by employing arbitrarily chosen integration limits and ignoring the elevated baseline to illustrate the method's intrinsic limits.

the present infrared data alone do not directly prove complexation between the sulfate groups of the surfactant and the ammonium groups of the polymer (macrophase-separated blends of polymer and surfactants would yield the same results), the occurrence of macrophase-separated polymer/surfactant blend can be ruled out since the precipitate has survived multiple washing steps in water, which would selectively remove unbound surfactant, thus supporting ionic complexation.

Attempts to establish more accurately the degree of loading, that is, the ratio between sulfate and ammonium groups, was done by performing NMR and elemental analysis on the complexes.

${ }^{1} \mathrm{H}$ and ${ }^{19} \mathrm{~F}$ NMR spectroscopy were performed on the three samples PG1-C12, PG2-C12, and PG3-C12, and elemental analysis was carried out on PG3-C12. On the basis of NMR spectroscopy alone, an accurate quantification could not be achieved. Unfavorable differences in line widths of the ${ }^{1} \mathrm{H}$ NMR signals of the dendronized polymers made comparative integrations unreliable. A typical example is shown in Figure 2 in which the expanded shift range from $\delta=2-5$ ppm of PG3C12 is depicted. This range contains the $\alpha-\mathrm{CH}_{2}$ signal of the surfactant at $\delta=3.65$ as well as the signal due to all $\mathrm{CH}_{2} \mathrm{O}$ groups of PG3 at $\delta=3.95 \mathrm{ppm}$, the intensity ratio of which describes the degree to which dodecylsulfate was incorporated as counterion in the polymer-lipid complex. Assuming a 100\% exchange, this ratio should be $16: 28$, respectively. As can be seen, depending on the integration limits chosen for the signal at $\delta=3.95 \mathrm{ppm}$, different ratios can be generated. On the basis of this method, it can therefore only be concluded that the level of exchange is considerable and may be close to or even above $80 \%$. The ${ }^{19} \mathrm{~F}$ NMR spectra of the complexes did not show any indication of residual trifluoroacetate. This cannot be interpreted as indication for a quantitative exchange, however, because, as mentioned in the preparation protocol, the solution of the dendronized polymers were acidified to $\mathrm{pH}=3-4$ prior to complexation using hydrochloric acid. It can therefore not be excluded that also some chlorides act as counterions for the ammoniums.

Most insight into the complexes composition came from elemental analysis. The values for the PG3-C12 complex are given in the following (element: calculated/found) whereby the calculated ones refer to $100 \%$ coverage and zero incorporation of chloride: C: $57.90 / 57.16 ; \mathrm{H}: \quad 8.69 / 8.71 ; \mathrm{N}: 4.98 / 5.02 ; \mathrm{S}$ : 6.51/6.56; Cl: $0.00 / 0.03$. These experimental values match the calculated ones almost perfectly so that a near quantitative exchange of trifluoroacetate against dodecylsulfate is indicated. It should be mentioned (a) that the sulfur value is large enough so as to detect an eventual lower coverage and (b) the chlorine value is so low so that chloride incorporation can practically be excluded.

3.2. Solid-State Characterization on Ionic Dendronized Polymer Complexes. Small- and wide-angle X-ray scattering combined with cross-polarized optical microscopy and scanning differential calorimetry allowed a detailed description of the structure of the dendronized polymer-lipid complexes obtained by changing the dendron generation and the surfactant tail length.

In order to illustrate the effect of alkyl tail length, Figure 3a reports representative DSC scans on dendronized polymer PG1 complexed with three lipids $\mathrm{C} 8, \mathrm{C} 12$, and $\mathrm{C} 18$, while Figure $3 \mathrm{~b}$ highlights the effect of dendron generation by showing the heat scan curves for PG1-C18, PG2-C18, and PG3-C18. The most important feature apparent from these DSC scans is that a pronounced endothermic fusion peak appeared systematically in systems complexed with $\mathrm{C} 18$. These peaks were centered at $65{ }^{\circ} \mathrm{C}$ for PG1-C18, $61{ }^{\circ} \mathrm{C}$ for PG2-C18, and $51{ }^{\circ} \mathrm{C}$ for PG3C18. They correspond in each case to the fusion of the crystalline alkyl tails, as also confirmed by the melting of crystalline peaks in the WAXS diffractograms. For both PG1C18 and PG2-C18 this peak was followed by a smaller endothermic one (more pronounced for PG1-C18), centered at $\sim 80{ }^{\circ} \mathrm{C}$, indicative of a first-order thermodynamic transition. This was consistent with birefringence which for both PG1C18 and PG2-C18 was observed up to $80^{\circ} \mathrm{C}$ and disappeared above this temperature. As will be shown later in this paper, the second endothermic peak in PG1-C18 and PG2-C18 correspond to the transition from a lamellar phase to an isotropic fluid. The complexation of PG3 with C18 leads to a decrease of the melting point of the lipid's crystalline phase and seems to suppress the second endothermic peak. Again, it will be shown that this is consistent with the lack of a stable lamellar phase for the PG3-C18 complex. Contrarily to the C18 case, no first-order thermodynamic transitions could be detected by DSC analysis in the complexes obtained with shorter alkyl tails. 

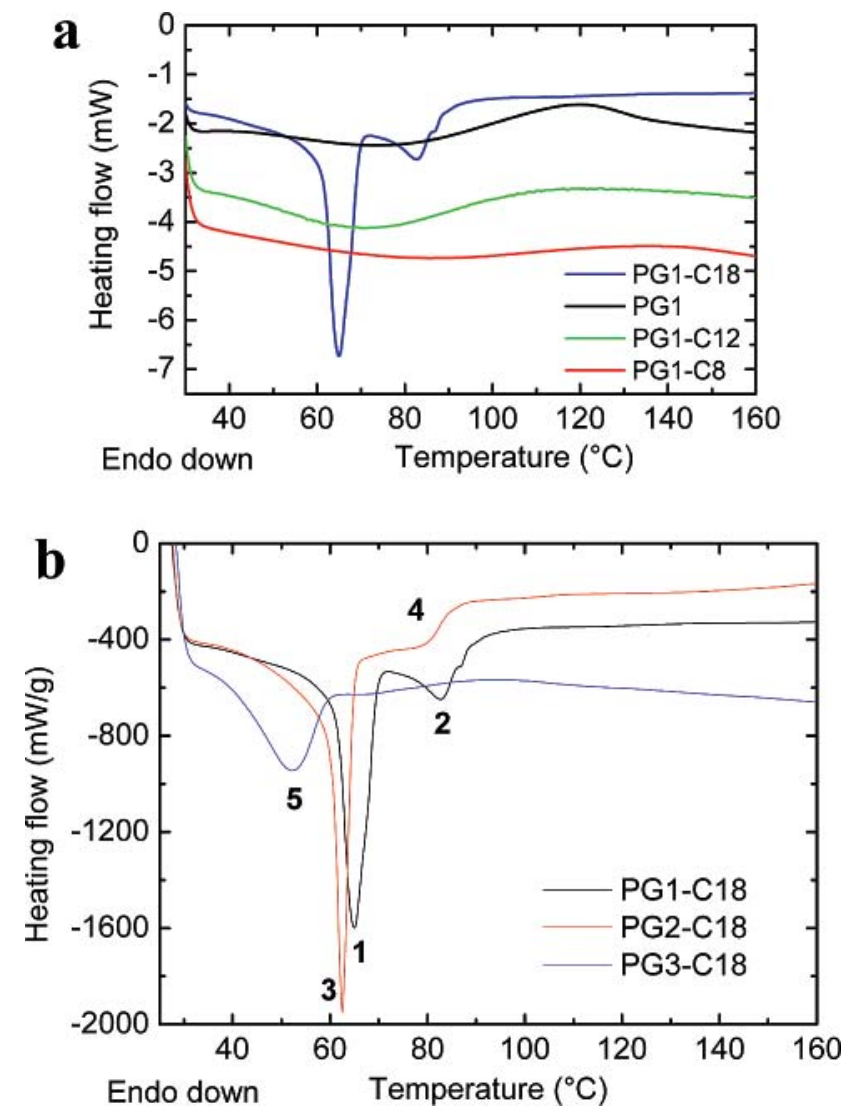

Figure 3. DSC scans at $10{ }^{\circ} \mathrm{C} / \mathrm{min}$ for the following annealed complexes: (a) PG1-C8 (amorphous), PG1-C12 (hexagonal phase), PG1-C18 (lamellar phase); (b) PG1-C18 (lamellar phase), PG2-C18 (lamellar phase), PG3-C18 (birefringent disordered phase). Enthalpies of transitions expressed as $\mathrm{kJ} / \mathrm{mol}$ of alkyl tails are 23.8, 5.1, 26.8, 2.4, and 18 for peaks 1 to 5 , respectively.

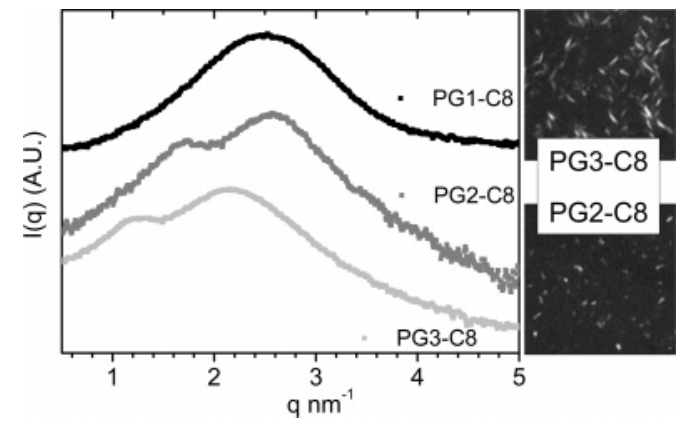

Figure 4. Solid-state SAXS diffractograms showing and isotropic homogeneous phase for PG1-C8 and disordered rectangular phases for PG2-C8 and PG3-C8. Cross-polarized optical microscopy pictures taken at room temperature for PG2-C8 and PG3-C8.

A better understanding and characterization of the liquidcrystalline phases could be gained by performing small-angle $\mathrm{X}$-ray scattering. Figure 4 shows the diffractograms measured for complexes formed by PG1, PG2, PG3, and C8 after annealing. In the case of PG1-C8, a single broad peak is present, indicating that the system is in an isotropic and amorphous state. This is consistent with cross-polarized optical microscopy, which shows no evidence of birefringence. In this case, the alkyl chains are too short to induce segregation between the polymer backboneand surfactant. The system is at equilibrium in an isotropic homogeneous phase, in which the average chain-to-chain distance can be calculated as $2 \pi / q^{*}=2.5 \mathrm{~nm}$, with $q^{*}$ being the $q$ position corresponding to the maximum intensity of the diffractogram. The value of $q^{*}$ is very close to that characteristic of the backbone-to-backbone distance ${ }^{37}$ previously reported for
PG1 alone, indicating that the surfactant is acting as a very short spacer among polymer chains, lubricating the chains and allowing an optimal packing of the complexes into a bulk isotropic phase.

Increasing the dendron generation leads to a change in the molecular organization of the complexes, as shown in Figure 4 by the diffractograms of PG2-C8 and PG3-C8, each characterized by two broad peaks, located at $q_{1}=1.7 \mathrm{~nm}^{-1}, q_{2}=2.6$ $\mathrm{nm}^{-1}$ and $q_{1}=1.2 \mathrm{~nm}^{-1}, q_{2}=2.12 \mathrm{~nm}^{-1}$, respectively. Figure 4 also highlights another difference compared to PG1-C8 complex, e.g., that PG2-C8 and PG3-C8 complexes are birefringent at room temperature, which is indicative of anisotropic materials. Considering the widths of the peaks, and since the spacing ratio between $q_{2} / q_{1}$ is not typical of any regular columnar or lamellar phase, the two peaks are attributed to the first two reflections $(h, k)=(10)$ and $(01)$ in poorly organized $P 2 m$ columnar rectangular liquid-crystalline phases. ${ }^{41}$ The lattice parameters $a$ and $b$ can be obtained by

$$
q=2 \pi \sqrt{\frac{h^{2}}{a^{2}}+\frac{k^{2}}{b^{2}}}
$$

This gives $(a ; b)$ lattice parameters of $(3.69 \mathrm{~nm} ; 2.41 \mathrm{~nm})$ and (5.23 nm; $2.96 \mathrm{~nm}$ ) for the PG2-C8 and PG3-C8, respectively. The systematic increase of the larger lattice parameter $a$ when going from PG1-C8 $\rightarrow$ PG2-C8 $\rightarrow$ PG3-C8 is consistent with the backbone-to-backbone distances reported for first-, second-, and third-generation dendronized polymers in bulk. ${ }^{33}$ Since the volume fraction of lipid is expected to decrease only weakly with generation when the lipid length is maintained constant and the generation is increased, this change must be essentially related to the change in possible conformations of the dendronized polymers. Furthermore, without precise estimates of volume fractions of the lipid and polymeric domains, it is impossible to predict whether the polymer and the alkyl tails occupy the continuous and the dispersed phase, respectively, in the PG2-C8 and PG3-C8 rectangular phases. Precise estimates of the volume fractions of lipid and polymer domains are possible, provided that both the degree of loading can be accurately determined together with the densities of both the dendronized polymers and alkyl tails. Work on this specific issue is in progress and will be reported in due course.

Increase of the alkyl tail length has more pronounced consequences on the volume fraction of the lipid phase and, consequently, on the driving force toward microphase segregation. This is highlighted in Figure 5a where the SAXS diffractograms for PG1-C12, PG2-C12, and PG3-C12 at room temperature are shown. In order to clearly show multiple reflections, the intensities are plotted as $I q^{2}$. The complex PG1C12 shows three Bragg peaks spaced as $1: \sqrt{ } 3: 2$, which is typical of a columnar hexagonal phase, as further supported by the birefringence pattern observed under cross polarized optical microscopy. Unlike columnar hexagonal phases obtained from diblock copolymers, the second peak is particularly weak in PG1-C12, which led the authors to attribute this complex to a lamellar phase in previous work. ${ }^{34}$ However, in plots of $I q^{2}$ vs $q$, this reflection appears systematically in each PG1-C12 sample. Since the $\sqrt{3}$ reflection in columnar hexagonal phases is vanishingly small as the volume fraction of cylinders approach 0.33, the weak reflection in PG1-C12 is possibly to be attributed to the high volume fraction of the cylinders, contrarily to the case of diblock copolymers, where the hexagonal phase is normally observed at a volume fraction of cylinders of typically 0.17 to 0.3 . In a columnar hexagonal phase, the distance between 

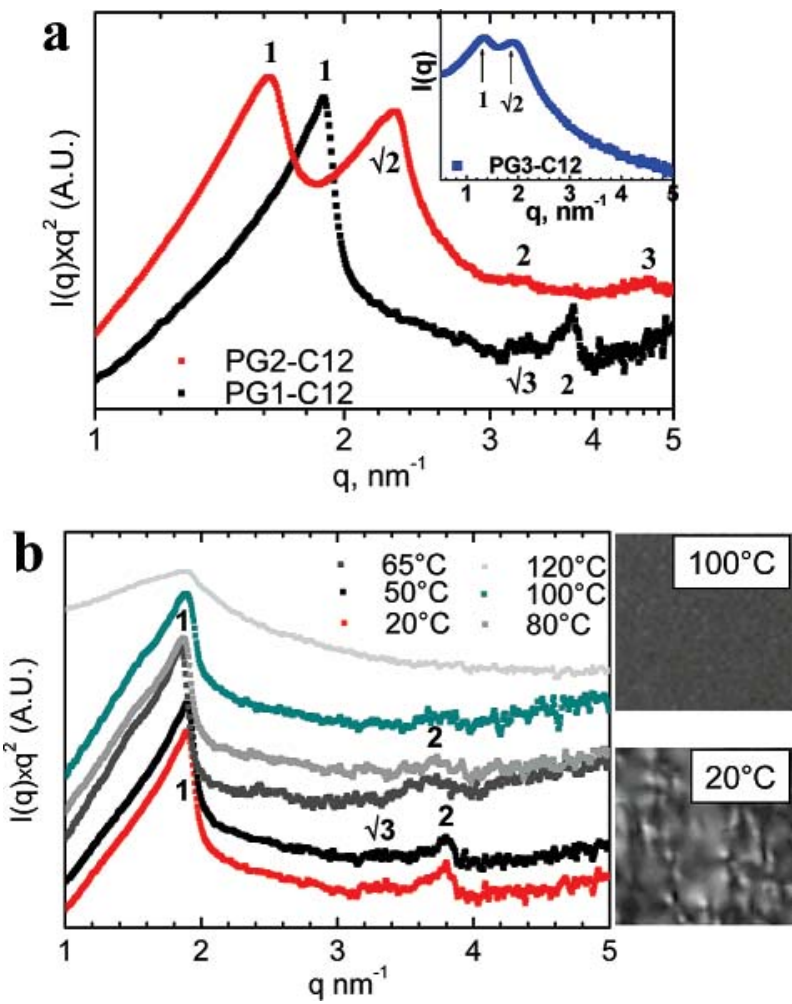

Figure 5. (a) SAXS diffractograms at room temperature plotted in $I(q)_{x} q^{2}$ vs $\log (q)$ to enhance the intensity of the higher order reflection peaks for the three liquid-crystalline phases obtained with C12: PG1C12 represents a columnar hexagonal phase; PG2-C12 and PG3-C12 (inset) represent a columnar tetragonal phase. (b) SAXS diffractogram of PG1-C12 signal plotted in $I(q)_{x} q^{2}$ vs $q$ at variable temperature from 20 to $120{ }^{\circ} \mathrm{C}$. Between 100 and $120^{\circ} \mathrm{C}$ the hexagonal phase melts into an isotropic fluid characterized by a broad peak, and the CPOM pictures show that the birefringence present at $20^{\circ} \mathrm{C}$ is lost at $100{ }^{\circ} \mathrm{C}$.

Bragg diffraction planes, $d_{h k l}$, is related to planes with Miller indexes $(h k 0)$ and to the lattice parameter $a$ by

$$
\frac{1}{d_{h k l}}=\sqrt{\frac{4}{3} \frac{h^{2}+h k+k^{2}}{a^{2}}}
$$

which yields $a$ directly by taking the distance $d_{h k l}=2 \pi / q_{1}$ for the reflection (100) with $q_{1}$ the $q$ value of the first reflection. In the case of PG1-C12 this yields a value of $3.85 \mathrm{~nm}$ for the lattice parameter, which is distinctly larger than the backboneto-backbone distance in PG1-C8 complexes, indicating a different organization of the polymer chains, as a result of the microsegregated morphology. By increasing temperature (Figure $5 \mathrm{~b}$ ), the hexagonal second and third reflections become progressively weaker, and above $100{ }^{\circ} \mathrm{C}$, when the birefringence of the sample gets lost, they disappear. At the same time, the first sharp peak changes into a broader one, indicating that the $T_{\text {ODT }}$ has been passed and that PG1-C12 is stable in an isotropic homogeneous fluid above $100{ }^{\circ} \mathrm{C}$.

When the generation is increased, the liquid crystals undergo further change in structure, as revealed by the diffractogram of PG2-C12 in Figure 5a. Four Bragg peaks spaced as $1: \sqrt{ } 2: 2: 3$ are visible, which are consistent with a tetragonal phase with long-range order.

In a columnar tetragonal phase the Bragg reflections and lattice parameter are related by

$$
q=2 \pi \sqrt{\frac{h^{2}}{a^{2}}+\frac{k^{2}}{a^{2}}}
$$

Equation 3 is a particular case of eq 1 with $a=b$. The four $1: \sqrt{ } 2: 2: 3$ Bragg peaks can then be identified with (100), (110), (200), and (300) reflections. Equation 1 identifies both centered columnar rectangular phases and simple columnar rectangular phase that are $C 2 / m$ and $P 2 m$ lattice spaces, respectively. The only difference in the Bragg spacing between $C 2 / m$ and $P 2 m$ is that the centered rectangular phase $C 2 / m$ should follow an extinction rule of the type $(h+k \neq 2 n+1)$. This is not consistent with the fourth (300) reflection observed in our case. Furthermore, when $a=b$, a centered tetragonal columnar phase with lattice period $a$ is equivalent to a simple tetragonal columnar phase with lattice period $a / \sqrt{ } 2$. Therefore, we can attribute unambiguously the PG2-C12 complex to a $P 2 m$ simple columnar tetragonal phase of lattice space $a=3.92 \mathrm{~nm}$, following eq 3 without further extinction rules. The reflection corresponding to (210) and (220) also characteristic of columnar tetragonal phases which for PG2-C12 are expected at 3.6 and $4.5 \mathrm{~nm}^{-1}$ are not visible in the diffractogramm of Figure $5 \mathrm{a}$ (though the (220) reflection can possibly be covered by the (300) reflection close by). It is illustrative to highlight the differences in lattice parameters between the PG2-C12 columnar tetragonal and the PG1-C12 columnar hexagonal phases. In the first case, the columnar tetragonal complex is obtained with a dendronized polymer of second generation and $\mathbf{C 1 2}$, while in the latter case, the columnar hexagonal phase is obtained with a dendronized polymer of first generation and C12. Despite the difference of one generation between the PG1 and PG2, only a difference of $\sim 0.1 \mathrm{~nm}$ is observed in the corresponding lattice spaces. This reflects a different molecular organization of the tetragonal and hexagonal phases which has to be related primarily to the different polymer backbone structures of PG1 and PG2 and possibly to a small decrease of the volume fraction of the alkyl tail when going from PG1-C12 to PG2-C12. On the other hand, the increase in alkyl tail lengths and volume fraction is considered to be the main cause driving the disordered rectangular PG2-C8 into a long-range ordered PG2-C12 phase. Also in this case, without exact determination of volume fractions of polymer and alkyl tail domains, and relying solely on SAXS, it is not possible to argue whether the lipid forms the cylinders or the continuous domains.

The PG2-C12 tetragonal phase proved to be stable for temperatures up to $170{ }^{\circ} \mathrm{C}$, maintaining both the same diffractogram and birefringence. Higher temperatures corresponded to the onset of thermal degradation of the complexes, as revealed by the yellowing of the complex powder.

When the generation was further increased to 3 and the length of the alkyl tail maintained at $\mathrm{C} 12$, only two Bragg reflections can be distinguished, spaced as $1: \sqrt{ } 2$. This is still consistent with a tetragonal phase, although the lack of further reflections indicates that the phase is poorly organized (inset in Figure 5a). The lattice parameter is measured at $4.72 \mathrm{~nm}$, which reflects the increase in generation of the polymer. As expected by the poor order, the PG3-C12 tetragonal phase was found to have a lower $T_{\mathrm{ODT}}$ compared to PG2-C12, with loss of birefringence at $90{ }^{\circ} \mathrm{C}$ and loss of Bragg reflections at $150{ }^{\circ} \mathrm{C}$.

By increasing the length of the alkyl tail to C14, the same liquid-crystalline structures found for the PG1-C12, PG2-C12, and PG3-C12 complexes were observed, as demonstrated by the SAXS diffractograms shown in Figure 6. The most surprising result is the size of the lattice parameter of the PG1-C14 hexagonal phase which went to $4.83 \mathrm{~nm}$ from the $3.85 \mathrm{~nm}$ of the PG1-C12 hexagonal phase. Moreover, the $T_{\text {ODT }}$ for PG1C14 was measured at $85{ }^{\circ} \mathrm{C}$, that is $15^{\circ} \mathrm{C}$ less than that measured for the PG1-C12 hexagonal phase. The increase in 


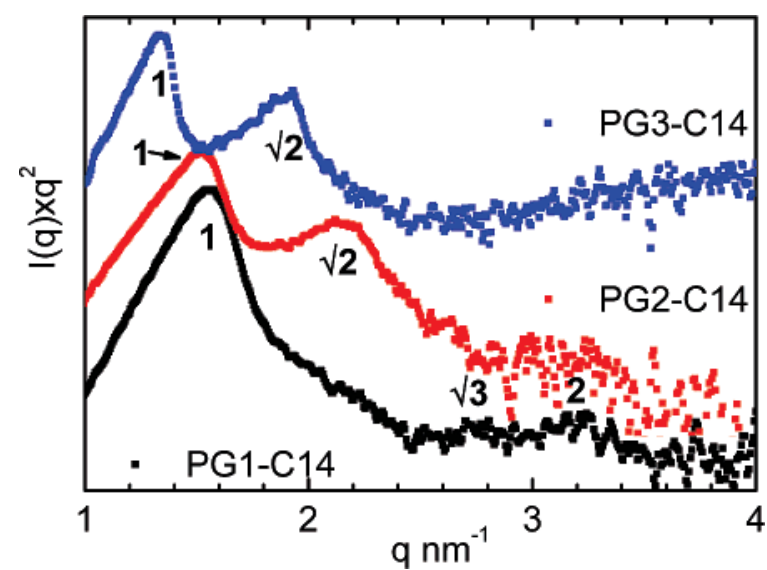

Figure 6. SAXS diffractograms plotted in $I(q)_{x} q^{2}$ vs $q$ for PG1-C14, PG2-C14, and PG3-C14 complexes at room temperature. For PG1C14, the signal is characteristic of an hexagonal columnar phase with weak second-order peak for the diffractograms of PG2-C14 and PG3C14 representing tetragonal columnar phases.

lattice parameter when going from PG1-C12 to PG1-C14 cannot be justified only by the increase in contour length of the surfactant $(0.26 \mathrm{~nm})$ and is more likely a consequence of the different packing of polymer and surfactant in the hexagonal phases. A tentative explanation of this increase will be given below. On the contrary, the columnar tetragonal phases observed for PG2-C14 and PG3-C14 were consistent with the homologous $\mathrm{C} 12$ series, with an increase in lattice parameter of 0.26 and $0.06 \mathrm{~nm}$, respectively.

When further increasing the volume fraction of the alkyl tail, by raising the length of the surfactant from $\mathrm{C} 14$ to $\mathrm{C} 18$, the structure of the liquid-crystalline phases obtained for the PG1C18, PG2-C18, and PG3-C18 complexes underwent further changes (Figure 7a). The diffractogram of PG1-C18 shows four Bragg peaks $q_{1}: q_{2}: q_{3}: q_{4}$ spaced as $1: 2: 3: 4$, which indicates a lamellar phase with long-range order and period $4.2 \mathrm{~nm}$. The PG2-C18 complex also shows a lamellar organization, with a period of $4.62 \mathrm{~nm}$. This is consistent with the increase of generation, although only two peaks characterize the diffractogram, indicating poorer ordering for the PG2-C18 phase compared to the PG1-C18 complex. SAXS diffractograms (Figure $7 \mathrm{~b}$ ) and Table 1 show that in both these complexes the lamellar phase clears at $65^{\circ} \mathrm{C}$, which is the melting temperature of the lipid chains, as discussed before. These data suggest that the lamellar phase is associated with crystalline alkyl tails and clears as soon as the lipid tails melt. Beyond $65{ }^{\circ} \mathrm{C}$, both the PG1-C18 and PG2-C18 are still birefringent, and their X-ray diffractogram at large angles (see inset in Figure $7 b$ ) highlights the fusion of the lipid crystalline sharp peak at $q=15.2 \mathrm{~nm}^{-1}$. Under these conditions, the lamellar order between polymerlipid interfaces is lost, but the interfaces are still prevalently aligned along a common director, as supported by the birefringence of the complexes. Finally, by further increasing temperature, to 80 and $100^{\circ} \mathrm{C}$, birefringence is lost for PG1-C18 and PG2-C18, respectively, and the SAXS diffractogram becomes characteristic of an isotropic, homogeneous fluid. Therefore, the second endothermic peak in the DSC scans of both PG1-C18 and PG2-C18 is consistent with the loss of birefringence and identifies a complete isotropization of the complexes. Accordingly, $65{ }^{\circ} \mathrm{C}$ can be identified as an order-nematic transition temperature and $80{ }^{\circ} \mathrm{C}$ (or $100{ }^{\circ} \mathrm{C}$ for PG2-C18) as a nematicisotropic transition. Similar thermotropic behavior has been reported for liquid-crystalline phases based on cationic hyperbranched polypeptides-lipid complexes. ${ }^{42}$
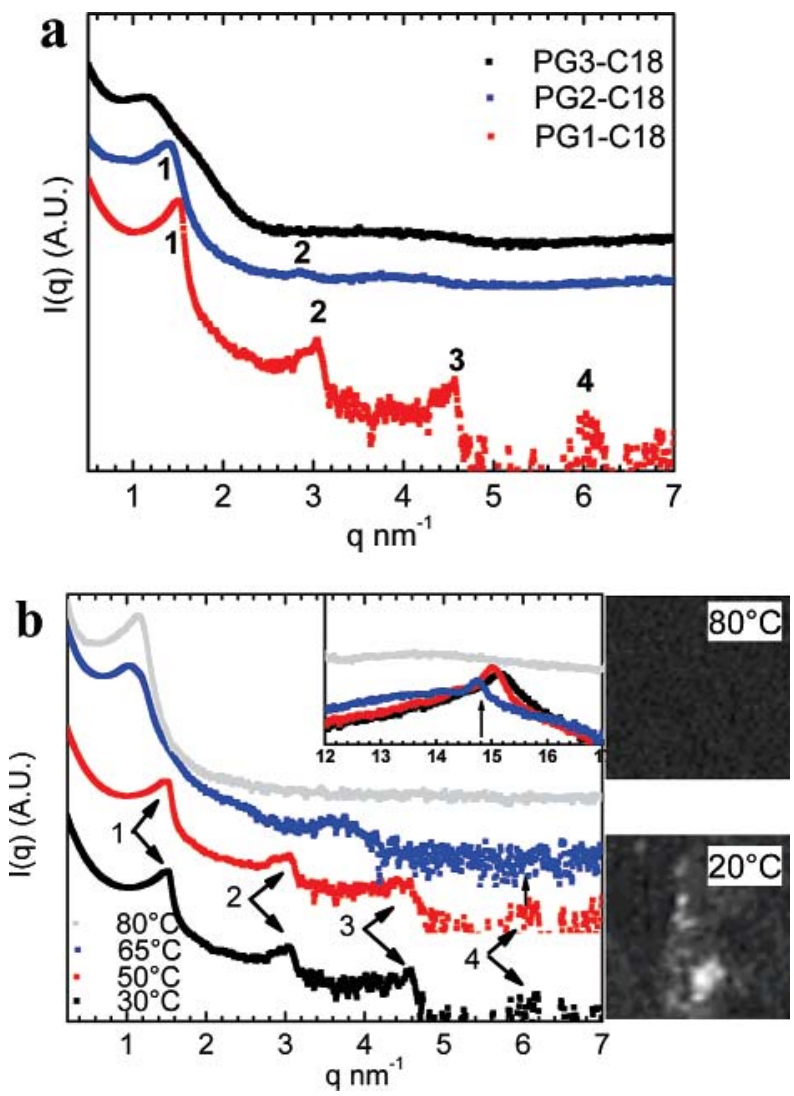

Figure 7. (a) SAXS diffractograms at room temperature of complexes formed by PG1, PG2, and PG3 complexed with C18 alkyl tails. Diffractograms indicate a lamellar phase for PG1-C18 and PG2-C18 complexes, while PG3-C18 presents a single peak accompanied by birefringence, which is not sufficient to attribute this phase. (b) Temperature-dependent SAXS diffractograms of PG1-C18 lamellar phase. Diffractograms show that the lamellar structure is cleared at $65^{\circ} \mathrm{C}$, although birefringence is present up to $80^{\circ} \mathrm{C}$. The inset shows that the characteristic peak of crystalline $\mathrm{C} 18$ lipid tails is cleared beyond $65^{\circ} \mathrm{C}$.

Differently from the previous two complexes, PG3-C18 is characterized by a broad peak at $1.2 \mathrm{~nm}^{-1}$, indicative of very poor order, although it shows birefringence, which indicates that the complex is organized into an anisotropic phase. However, when the temperature is increased over the melting point of the lipids and the glass transition of the polymer, the complex starts to organize into a more ordered structure. This is revealed by the sharpening of the first peak and appearance of a second shoulder, spaced as $q_{1}: q_{2}$ equal to $1: 2$, which suggests formation of lamellar phase (Figure 8). Upon cooling back to room temperature, the double peak with 1:2 spacing is maintained. Presumably, the rigidity of the PG3 chain, combined with the crystallinity of $\mathrm{C} 18$, prevents the complex from forming a wellorganized liquid-crystalline phase at low temperature, whereas, upon temperature increase, the complex gains progressively mobility and starts to organize into a microphase-separated complex fluid.

Based on the results discussed, the phase diagram with orderdisorder transition temperatures is shown in Figure 9. For all complexes investigated the temperature windows are reported, for which stable organized liquid-crystalline phases, anisotropic fluids, and isotropic homogeneous fluids are found (see also Table 1). The state diagram shown in Figure 10 reports the liquid-crystalline phases thermodynamically stable at room temperature for all the dendronized polymer-lipid complexes studied. By comparing the PG1-C12, PG1-C14, and PG1-C18 structures in Figure 10, a tentative explanation of the increase 
Table 1. Temperatures (in ${ }^{\circ} \mathrm{C}$ ) Indicating the Order-Disorder Transition Temperatures As Measured by SAXS and the Loss of Birefringence under Cross-Polarized Optical Microscopy (CPOM)

\begin{tabular}{|c|c|c|c|c|c|c|c|c|c|c|c|}
\hline & \multicolumn{3}{|c|}{ PG1 } & \multicolumn{4}{|c|}{ PG2 } & \multicolumn{4}{|c|}{ PG3 } \\
\hline & $\mathrm{C} 12$ & C14 & $\mathrm{C} 18$ & $\mathrm{C} 8$ & $\mathrm{C} 12$ & $\mathrm{C} 14$ & $\mathrm{C} 18$ & $\mathrm{C} 8$ & $\mathrm{C} 12$ & C14 & $\mathrm{C} 18$ \\
\hline SAXS & 100 & 85 & 65 & $150<T^{\circ}<170$ & 160 & $150<T^{\circ}<170$ & 65 & 170 & 150 & 160 & $>170$ \\
\hline CPOM & 100 & & $80^{a}$ & 105 & 150 & & $100^{a}$ & 75 & 90 & & 120 \\
\hline
\end{tabular}

${ }^{a}$ Indicates a nematic-isotropic transition.

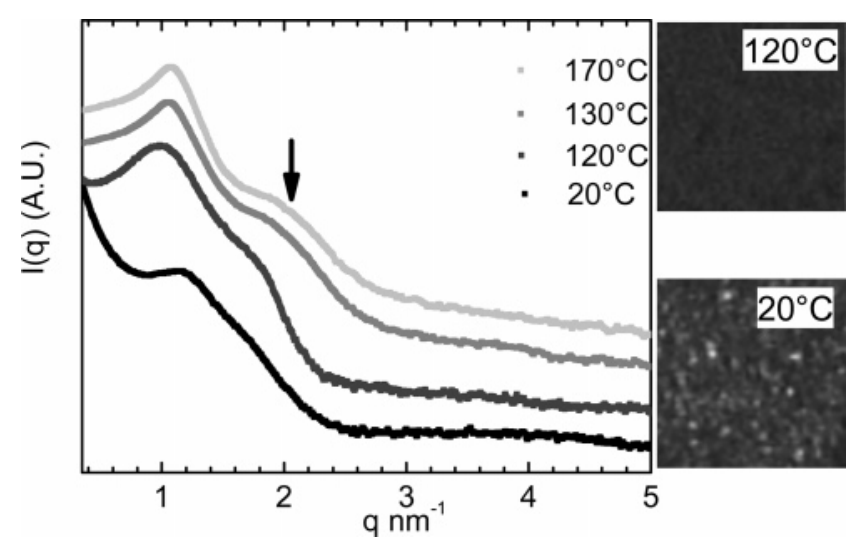

Figure 8. Temperature-dependent SAXS diffractograms of PG3-C18 at various temperatures. At $120{ }^{\circ} \mathrm{C}$ a shoulder centered at $q_{2}=2 \mathrm{~nm}^{-1}$ appears $\left(q_{2} \approx 2 q_{1}\right)$ and becomes more pronounced while increasing temperature up to $170{ }^{\circ} \mathrm{C}$. The birefringence is lost at $120^{\circ} \mathrm{C}$.

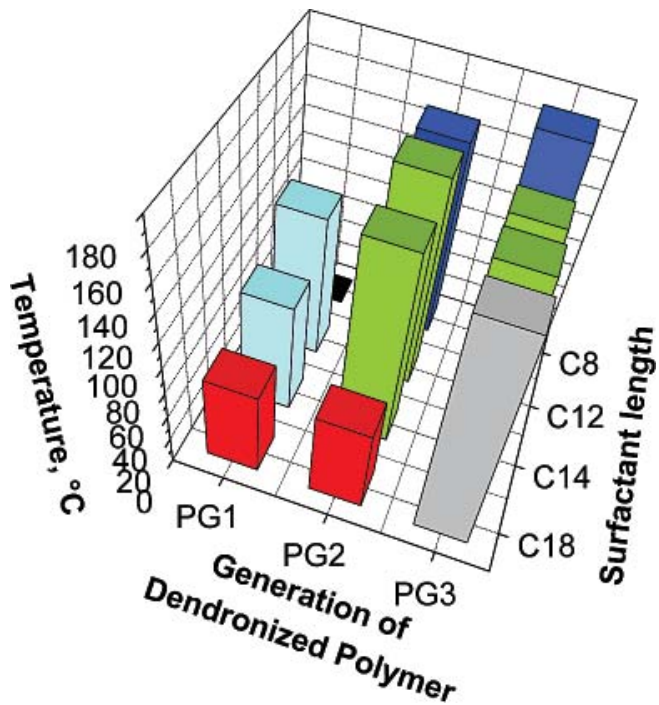

Figure 9. Phase diagram summarizing the transition temperatures observed by SAXS for the complexes investigated: PG1-C8 (amorphous, black), [PG1-C12, PG1-C14] (columnar hexagonal phases, light blue), [PG1-C18, PG2-C18] (lamellar phases, red), [PG2-C8, PG3C8] (rectangular phases, dark blue), [PG2-C12, PG2-C14, PG3-C12, PG3-C14] (columnar tetragonal phases, light green), PG3-C18 (birefringent disordered phase, gray).

in lattice parameter of $1 \mathrm{~nm}$ when going from PG1-C12 to PG1C14 can be formulated. When the length of the alkyl tail is increased by two units, while remaining in a hexagonal phase, the PG1-C14 complex starts to approach the order-to-order hexagonal-lamellar transition and is thus characterized by different molecular packing compared to hexagonal phases far apart from phase boundaries (PG1-C12). Eventually, by further increasing the length of the alkyl tail, contiguous cylinders start to merge, leading to a lamellar phase (PG1-C18). This is consistent with the projection of the lattice parameter of PG1C14, $a=4.83 \mathrm{~nm}$, along the direction orthogonal to the planes formed by merging cylinders. The lattice parameter arising from this projection is $a \cos \left(30^{\circ}\right)=4.18 \mathrm{~nm}$, and this value closely

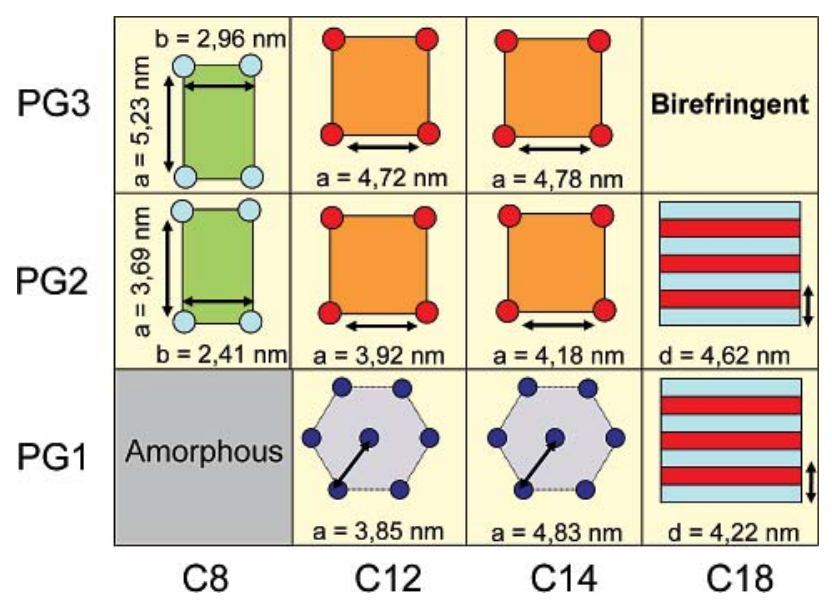

Figure 10. State diagram summarizing the liquid-crystalline phases stable at room temperature for the complexes investigated. For each phase, the lattice parameters determined by small-angle X-ray scattering are provided.

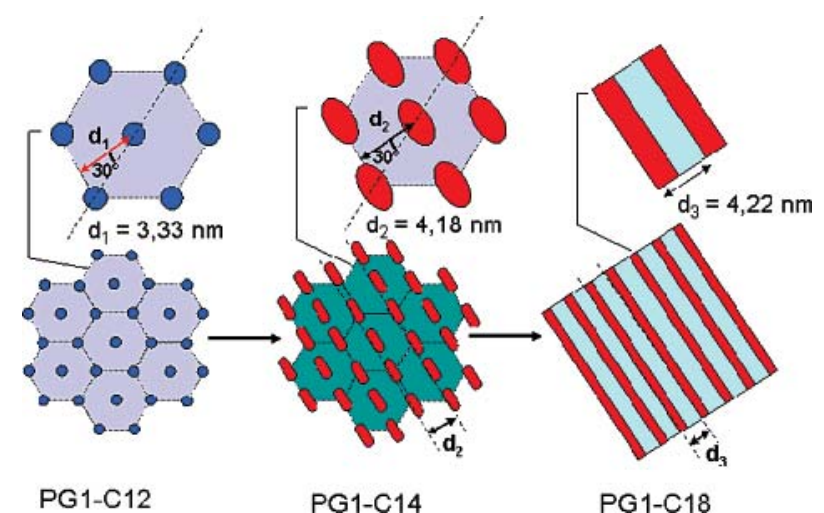

Figure 11. Sketch of the transition from the columnar hexagonal phase (PG1-C12 and PG1-C14) to a lamellar phase (PG1-C18).

matches the lamellar spacing of PG1-C18. Figure 11 illustrates schematically the transition from PG1-C14 hexagonal to PG1C18 lamellar.

\section{Conclusions}

We have described the liquid-crystalline (LC) behavior of supramolecular assemblies between cationic dendronized polymers from generation 1 to 3 (PG1-PG3) and anionic surfactants of four different hydrocarbon lengths, by combining small-angle $\mathrm{X}$-ray scattering, differential scanning calorimetry, and cross polarized optical microscopy.

At short alkyl tail lengths (C8) either isotropic homogeneous phases or short-range ordered rectangular columnar phases were observed for the complexes, as the alkyl tail was too short to induce long-range ordered liquid-crystalline structures. However, by increasing the alkyl surfactant chain to $\mathrm{C} 12$, both columnar hexagonal (for PG1) and columnar tetragonal phases (PG2 and PG3) could be observed, as a result of the increased segregation between the alkyl tails and the polymer backbone. Similar results were obtained by increasing the length of the alkyl tail to C14, 
with a consistent increase in the lattice parameter of the LC phases as a result of the increased length of the alkyl tail. In the case of PG1-C14, an increase of $1 \mathrm{~nm}$ with respect to the PG1-C12 hexagonal phase was interpreted by the approaching of the hexagonal-lamellar transition. When the length of the alkyl tails was increased to $\mathrm{C} 18$, a lamellar phase with crystalline lipid domains was observed for both the PG1-C18 and PG2C18 complexes, while the PG3-C18 complex remained, at room temperature, stable as a birefringent disordered phase. Upon temperature increase, the lamellar phase of PG1-C18 and PG2C18 complexes was rapidly lost as soon as the alkyl lipid tails were molten, while the PG3-C18 complex relaxed to a more organized phase (with a second reflection appearing at $2 q^{*}$ ), presumably due to an increased mobility of both dendronized polymer and alkyl tail chains. By combining temperaturedependent small-angle X-ray scattering and cross-polarized optical microscopy for the other phases, it could be established that the columnar tetragonal phases have very high orderdisorder transition temperatures (above $170{ }^{\circ} \mathrm{C}$ ), while the hexagonal columnar phases undergo an order-disorder transition at 85 or $100{ }^{\circ} \mathrm{C}$, depending on the length of the alkyl tail. From this study, it appears evident that phase changes occur within small changes of volume fractions compared to block copolymer phase diagrams and that order-order transitions are induced essentially by the length of the lipid tails and the generation of dendronized polymers. While these findings encourage further studies to assess more precisely the effects of the volume fraction and molecular packing of polymer and lipid chains on the phase diagram, they also provide a solid framework for designing various types of liquid-crystalline polymeric structures and finely controlling their characteristic periodic size within a few nanometers typical length scale. In particular, the various types of columnar phases found in this work could be used as templates for mesopourous materials and functional membranes where surfaces can be charged in a $\mathrm{pH}-$ responsive way and pores controlled in size, packing, and orientation.

Acknowledgment. We are grateful to Dr. Jean Nicolas Aebischer of the Engineer School of Fribourg for allowing access to their differential scanning calorimetry and infrared spectroscopy equipment. Prof. Janne Ruokolainen, Helsinki University of Technology, is acknowledged for fruitful discussions. The Swiss National Science Foundation is gratefully acknowledged for financial support. We cordially thank Dr. H. Rueegger, M. Schneider, and M. Colussi, all at ETHZ, for their competent help with NMR spectroscopical, elemetal analysis, and GPC, DSC, and TGA measurements, respectively.

\section{References and Notes}

(1) Leibler, L. Macromolecules 1980, 13, 1602-1617.

(2) Matsen, M. W.; Bates, F. S. Macromolecules 1996, 29, 1091-1098.

(3) Bates, F. S.; Fredrickson, G. H. Annu. Rev. Phys. Chem. 1990, 41, $525-557$.
(4) Stadler, R.; Auschra, C.; Beckmann, J.; Krappe, U.; Voigt-Martin, I.; Leibler, L. Macromolecules 1995, 28, 3080-3097.

(5) Brinkmann, S.; Stadler, R.; Thomas, E. L. Macromolecules 1998, 31 , 6566-6572.

(6) Lee, M.; Cho, B. K.; Zin, W. C. Chem. Rev. 2001, 101, 3869-3892.

(7) Olsen, B. D.; Segalman, R. A. Macromolecules 2005, 38, 1012710137.

(8) Epps, T. H.; Bates, F. S. Macromolecules 2006, 39, 2676-2682.

(9) Fischer, H.; Poser, S.; Arnold, M.; Frankt, W. Macromolecules 1994, 27, 7133-7138.

(10) Gallot, B. Prog. Polym. Sci. 1996, 21, 1035-1088.

(11) Mao, G.; Wang, J.; Clingman, S. R.; Ober, C. K.; Chen, J. T.; Thomas, E. L. Macromolecules 1997, 30, 2556-2567.

(12) Nap, R. J.; ten Brinke, G. Macromolecules 2002, 35, 952-959.

(13) Schlaada, H.; Kukulaa, H.; Smarslya, B.; Antonietti, M.; Pakula, T. Polymer 2002, 43, 5321-5328.

(14) Ganicz, T.; Pakula, T.; Fortuniak, W.; Bialecka-Florjanczyk, E. Polymer 2005, 46, 11380-11388.

(15) Bauer, S.; Ringsdorf, H.; Fischer, H. Angew. Chem., Int. Ed. 1993 $32,1589-1592$

(16) Lorenz, K.; Hölter, D.; Stühn, B.; Mülhaupt, R.; Frey, H. Adv. Mater. 1996, 8, 414-416.

(17) Percec, V.; Johansson, G.; Ungar, G.; Zhou, J. J. Am. Chem. Soc. 1996, 118, 9855-9866.

(18) Pesak, D. J.; Moore, J. S. Angew. Chem., Int. Ed. 1997, 36, 16361639.

(19) Percec, V.; Cho, W.-D.; Moller, M.; Prokhorova, S. A.; Ungar, G.; Yeardley, D. J. P. J. Am. Chem. Soc. 2000, 122, 4249-4250.

(20) Park, C.; Choi, K. S.; Song, Y.; Jeon, H.-J.; Song, H. H.; Chang, J. Y.; Kim, C. Langmuir 2006, 22, 3812-3817.

(21) Ponomarenko, S. A.; Boiko, N. I.; Shibaev, V. P.; Richardson, R. M.; Whitehouse, I. J.; Rebrov, E. A.; Muzafarov, A. M. Macromolecules 2000, 33, 5549-5558.

(22) Ruokolainen, J.; ten Brinke, G.; Ikkala, O. Adv. Mater. 1999, 11, 777780.

(23) Valkama, S.; Lehtonen, O.; Lappalainen, K.; Harri Kosonen, H.; Castro, P.; Repo, T.; Torkkeli, M.; Serimaa, R.; ten Brinke, G.; Leskelä, M.; Ikkala, O. Macromol. Rapid Commun. 2003, 24, 556-560.

(24) Kato, T. Science 2002, 295, 2414-2418.

(25) Kato, T.; Mizoshita, N.; Kishimoto, K. Angew. Chem., Int. Ed. 2006 , $45,38-68$.

(26) ten Brinke, G.; Ikkala, O. Chem. Rec. 2004, 4, 219-230.

(27) Antonietti, M.; Conrad, J.; Thunemann, A. Macromolecules 1994, 27, $6007-6011$

(28) Antonietti, M.; Burger, C.; Effing, J. Adv. Mater. 1995, 7, 751-753.

(29) Guan, Y.; Antonietti, M.; Faul, C. F. J. Langmuir 2002, 18, 59395945.

(30) Martin-Rapun, R.; Marcos, M.; Omenat, A.; Barbera, J.; Romero, P.; Serrano, J. L. J. Am. Chem. Soc. 2005, 127, 7397-7403.

(31) Ringsdorf, H.; Schlard, B.; Venzmer, J. Angew. Chem., Int. Ed. 1988, $27,113-158$

(32) Schlüter, A. D.; Rabe, J. P. Angew. Chem., Int. Ed. 2000, 39, 864883.

(33) Zhang, A.; Shu, L.; Bo, Z.; Schlüter, A. D. Macromol. Chem. Phys. 2003, 204, 328-339.

(34) Canilho, N.; Kasëmi, E.; Mezzenga, R.; Schlüter, A. D. J. Am. Chem. Soc. 2006, 128, 13998-13999.

(35) Schlüter, A. D. Top. Curr. Chem. 2005, 151-191.

(36) Zhang, A.; Zhang, B.; Wächtersbach, E.; Schmidt, M.; Schlüter, A. D. Chem.-Eur. J. 2003, 9, 6083-6092.

(37) Zhang, A.; Okrasa, L.; Pakula, T.; Schlüter, A. D. J. Am. Chem. Soc. 2004, 126, 6658-6666.

(38) Kasëmi, E.; Zhuang, W.; Rabe, J. P.; Fischer, K.; Schmidt, M.; Colussi, M.; Keul, H.; Yi, D.; Colfen, H.; Schlüter, A. D. J. Am. Chem. Soc. 2006, 128, 5091-5099.

(39) Quina, F. H.; Nassar, P. M.; Bonilha, J. B. S.; Bales, B. L. J. Phys. Chem. 1995, 99, 17028-17031.

(40) Goddard, E. D. Colloids Surf. 1986, 19, 301-329.

(41) Komatsu, T.; Ohta, K.; Watanabe, T.; Ikemoto, H.; Fujimoto, T.; Yamamoto, I. J. Mater. Chem. 1994, 4, 537-540.

(42) Canilho, N.; Scholl, M.; Mezzenga, R.; Klok, A.-H., to be submitted for publication. 


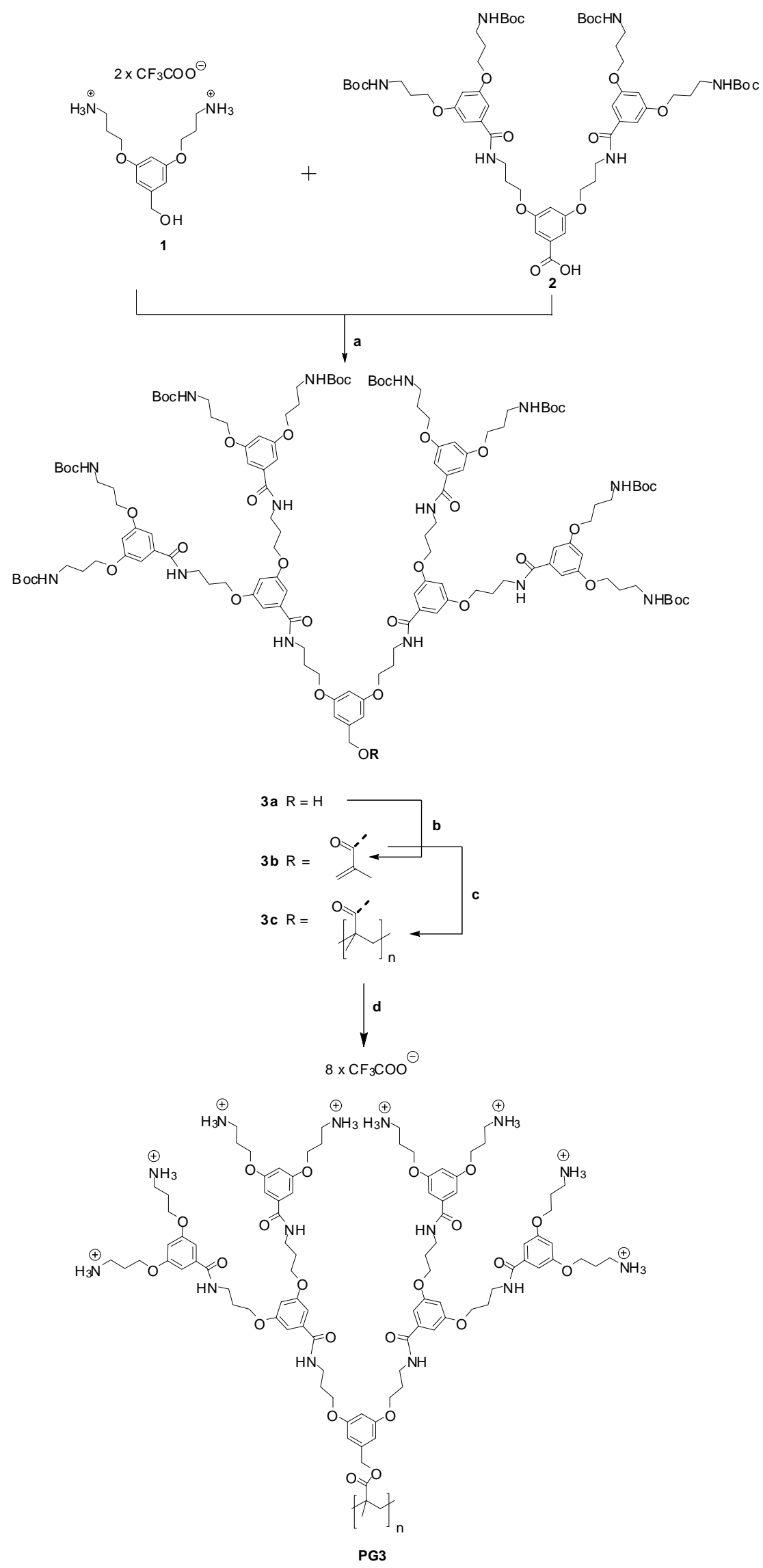

Scheme 1. Reagents and conditions: a) HOBt, EDC, DMF, $-20^{\circ} \mathrm{C}, 16 \mathrm{~h}(67 \%)$; b) MAC, DMAP, DMF, RT, 16 h (81\%) ; c) AIBN, DMF, 65ㅇ , 16 h (65\%) ; d) 3c, TFA, RT, 48 h (85\%) 


\section{Synthesis:}

General: Compounds $\mathbf{1}^{31}$ and $\mathbf{2}^{35}$ were synthesized according to literature methods. Other reagents were purchased from Aldrich, Across or Fluka. Methacryloyl chloride (MAC) was freshly distilled before use.All other reagents and solvents were used as received. All reactions were performed under nitrogen atmosphere. Silica gel $60 \mathrm{M}$ (Macherey-Nagel, 0.04-0.063 mm/230400 mesh) was used as the stationary phase for column chromatography. Whenever possible, reactions were monitored by thin-layer chromatography (TLC) using TLC silica gel coated aluminum plates $60 \mathrm{~F}_{254}$ (Merck). Compounds were detected by UV light (254 nm or $366 \mathrm{~nm}$ ) and/or by treatment with a solution of ninhydrine in ethanol followed by heating. If not otherwise noted, ${ }^{1} \mathrm{H}$ and ${ }^{13} \mathrm{C}$ NMR spectra were recorded on Bruker AM 300 $\left({ }^{1} \mathrm{H}: 300 \mathrm{MHz},{ }^{13} \mathrm{C}: 75 \mathrm{MHz}\right)$ and AV $500\left({ }^{1} \mathrm{H}: 500 \mathrm{MHz},{ }^{13} \mathrm{C}: 125 \mathrm{MHz}\right)$ spectrometers at room temperature using chloroform-d as a solvent. ${ }^{19} \mathrm{~F}$ NMR spectra were done on Bruker AM $200\left({ }^{19} \mathrm{~F}: 188 \mathrm{MHz}\right)$. High-resolution mass spectral (HRMS) were performed by the MS-service of the Laboratorium für Organische Chemie at ETH Zürich. MALDI-MS were run on an IonSpec Ultra instrument where 2-[(2E)-(4-tertbutylphenyl)-2-methylprop-2-enylidene]malononitrile (DCTB) or 3-hydroxypyridine 2-carboxylic acid (3-HPA) served as the matrix. Elemental analyses were performed by the Mikrolabor of the Laboratorium für Organische Chemie, ETH Zürich. The samples were dried rigorously under vacuum prior to analysis to remove strongly adhering solvent molecules. Gel permeation chromatography (GPC) measurements were carried out using PL-GPC 220 instrument with 2x PL-Gel Mix-B LS column set equipped with RI (refractive index), viscosity and LS (Light Scattering with $15^{\circ}$ and $90^{\circ}$ angle) detectors [DMF $+1 \mathrm{gL}^{-1} \mathrm{LiBr}$ as eluent at $80^{\circ} \mathrm{C}$ ]. Universal calibration was done using PMMA standards in a range of $M_{p}=2,680$ to 3,900,000 (Polymer Labs. Ltd, UK). 


\section{3,5-Bis(3-(3,5-bis(3-(3,5-bis(3-(tert-butoxycarbonylamino)propoxy) benzamido)propoxy)benzamido)propoxy)benzyl alcohol (3a)}

$\mathrm{N}$-Hydroxybenzotriazole $(1.12 \mathrm{~g}, 8.3 \mathrm{mmol})$ was added to a solution of acid 2 $(8.1 \mathrm{~g}, 6.92 \mathrm{mmol})$ in dry DMF $(150 \mathrm{~mL})$ at room temperature. After $10 \mathrm{~min} \mathrm{~N}$ (3-dimethylaminopropyl)-N'-ethylcarbodiimide hydrochloride (1.75 g, 9.13 $\mathrm{mmol}$ ) was added at $-20^{\circ} \mathrm{C}$, and the reaction mixture was stirred until the hydrochloride was dissolved completely (ca. $4 \mathrm{~h}$ ). Then a solution of TEA (3.30 g, $33 \mathrm{mmol})$ and 1 (1.6 g, $3.3 \mathrm{mmol})$ in methanol/DMF (10 mL, 1/ 1) was added dropwise at $-10^{\circ} \mathrm{C}$. The resulting mixture was warmed to room temperature and stirred for $16 \mathrm{~h}$. Solvent was removed in vacuo and residue again dissolved in $\mathrm{DCM}$ and then washed with aqueous $\mathrm{NaHCO}_{3}$ and brine. The organic layer was dried with magnesium sulphate and the solvent removed in vacuo. Two successive chromatographic separations (silica gel, $\mathrm{DCM}$ /methanol 20/1) and ethyl acetate/methanol 15/1) yielded $3 \mathrm{a}$ as a colorless foam (5.70 g, $67 \%) ; R_{f}=0.38(10: 1 \mathrm{DCM} / \mathrm{MeOH}) ;$ M.p. $=97-99$ ${ }^{\circ} \mathrm{C}$; ${ }^{1} \mathrm{H}$ NMR $\left(\mathrm{CDCl}_{3} / \mathrm{CD}_{3} \mathrm{OD}\right): \delta=1.42\left(\mathrm{~s}, 72 \mathrm{H}\right.$; $\left.{ }^{\mathrm{t}} \mathrm{Bu}\right), 1.93(\mathrm{~m}, 16 \mathrm{H}$; $\left.\mathrm{OCH}_{2} \mathrm{C}_{2} \mathrm{CH}_{2} \mathrm{~N}\right), 2.08\left(\mathrm{~m}, 12 \mathrm{H} ; \mathrm{OCH}_{2} \mathrm{C}_{2} \mathrm{CH}_{2} \mathrm{~N}\right), 3.25$ (t, $\left.16 \mathrm{H} ; \mathrm{CH}_{2} \mathrm{NH}\right), 3.55$ (t, $12 \mathrm{H} ; \mathrm{CH}_{2} \mathrm{NH}$ ), 3.99 (t, $16 \mathrm{H}$; $\mathrm{PhOCH}_{2}$ ), 4.03 (t, $12 \mathrm{H} ; \mathrm{PhOCH}_{2}$ ), 4.55 (s, 2 $\mathrm{H}$; $\left.\mathrm{CH}_{2}\right), 5.70(\mathrm{br}, 1 \mathrm{H}, \mathrm{OH}), 6.33(\mathrm{t}, 1 \mathrm{H} ; \mathrm{Ph}), 6.51(\mathrm{~d}, 2 \mathrm{H} ; \mathrm{Ph}), 6.55(\mathrm{t}, 4 \mathrm{H}$, $\mathrm{Ph}), 6.94\left(\mathrm{~d}, 12 \mathrm{H}\right.$; Ph), 7.92, 7.98 (br, $14 \mathrm{H}$; NH) ppm; ${ }^{13} \mathrm{C} N \mathrm{NMR}$ $\left(\mathrm{CDCl}_{3} / \mathrm{CD}_{3} \mathrm{OD}\right): \delta=28.47\left(\mathrm{C}\left(\underline{\mathrm{CH}}_{3}\right)_{3}\right), 29.04,29.14,29.62\left(\mathrm{OCH}_{2} \underline{\mathrm{CH}}_{2} \mathrm{CH}_{2} \mathrm{~N}\right)$, 37.71, 37.94, $38.07\left(\mathrm{OCH}_{2} \mathrm{CH}_{2} \underline{\mathrm{CH}}_{2} \mathrm{~N}\right), 64.43\left(\mathrm{OCH}_{2}\right), 66.09,66.43,66.51$ $\left(\mathrm{O}_{\underline{C}} \mathrm{H}_{2} \mathrm{CH}_{2} \mathrm{CH}_{2} \mathrm{~N}\right), 79.56\left(\underline{\mathrm{C}}\left(\mathrm{CH}_{3}\right)_{3}\right), 100.68,104.90,105.02,105.57,106.04$, 136.60, 136.70, 144.13, 144.21, 160.14, 160.21, 160.24 (Ar), 156.97, 168.38, 168.49 (NHCOO) ppm; HRMS-MALDI: $m / z$ (rel-\%): 2457.34 (65) [M Boc + Na $]^{+}, 2579.37(100)\left[\mathrm{M}+\mathrm{Na}^{+}, 2595.34\right.$ (10) $[\mathrm{M}+\mathrm{K}]^{+}$; elemental analysis (\%) calcd. for $\mathrm{C}_{131} \mathrm{H}_{194} \mathrm{~N}_{14} \mathrm{O}_{37}$ (2557.01): C 61.53, H 7.65, N 7.67; found: C 61.33, H 7.60, N 7.61. 


\section{3,5-Bis(3-(3,5-bis(3-(3,5-bis(3-(tert-butoxycarbonylamino)propoxy) benzamido)propoxy)benzamido)propoxy)benzyl methacrylate (3b)}

A solution of MAC $(0.44 \mathrm{~g}, 4.46 \mathrm{mmol})$ in DMF $(10 \mathrm{~mL})$ was added dropwise to a mixture of $3 \mathrm{a}(5.70 \mathrm{~g}, 2.23 \mathrm{mmol})$, triethylamine (TEA; $0.67 \mathrm{~g}, 6.70 \mathrm{mmol}$ ), and DMAP $(250 \mathrm{mg})$ in dry DMF $(100 \mathrm{~mL})$ at $0^{\circ} \mathrm{C}$ over $30 \mathrm{~min}$. The resulting mixture was warmed to room temperature and stirred for $16 \mathrm{~h}$. Solvent was removed in vacuo and residue again dissolved in DCM and then washed with aqueous $\mathrm{NaHCO}_{3}$ and brine. The organic layer was dried with magnesium sulphate and the solvent removed in vacuo. Three successive chromatographic separations (silica gel, DCM/methanol 20/1 and ethyl acetate/methanol 10/1) yielded $\mathbf{3 b}$ as colorless foam (4.67 g, $81 \%) ; R_{f}=0.68$ (DCM/Methanol 10/1); M.p. $=99-101^{\circ} \mathrm{C} ;{ }^{1} \mathrm{H} \mathrm{NMR}\left(\mathrm{CDCl}_{3}\right): \delta=1.42(\mathrm{~s}, 72 \mathrm{H}$; $\left.{ }^{t} \mathrm{Bu}\right), 1.87\left(\mathrm{~m}, 19 \mathrm{H} ; \mathrm{C}_{2}+\mathrm{C}=\mathrm{CC} \underline{H}_{3}\right), 2.03\left(\mathrm{~m}, 12 \mathrm{H} ; \mathrm{CH}_{2}\right), 3.20(\mathrm{~m}, 16 \mathrm{H}$; $\left.\mathrm{C}_{2} \mathrm{NH}\right), 3.52\left(\mathrm{~m}, 12 \mathrm{H} ; \underline{\mathrm{C}}_{2} \mathrm{NH}\right), 3.89\left(\mathrm{~m}, 16 \mathrm{H} ; \mathrm{PhOCH}_{2}\right), 4.00(\mathrm{~m}, 12 \mathrm{H}$; $\left.\mathrm{PhOCH}_{2}\right), 5.01\left(\mathrm{~s}, 2 \mathrm{H}\right.$; $\left.\mathrm{OCH}_{2} \mathrm{Ph}\right), 5.42\left(\mathrm{~m}, 1 \mathrm{H} ; \mathrm{C}=\mathrm{CH}_{2}\right), 6.09(\mathrm{~s}, 1 \mathrm{H}$; $\left.\mathrm{C}=\mathrm{CH}_{2}\right), 6.36(\mathrm{t}, 1 \mathrm{H} ; \mathrm{Ph}), 6.80(\mathrm{~d}, 2 \mathrm{H} ; \mathrm{Ph}), 6.44(\mathrm{t}, 4 \mathrm{H}, \mathrm{Ph}), 6.88(\mathrm{~d}, 12 \mathrm{H}$; $\mathrm{Ph}), 7.54(\mathrm{br}, 14, \mathrm{NH}) \mathrm{ppm} ;{ }^{13} \mathrm{C} \mathrm{NMR}\left(\mathrm{CDCl}_{3}\right): \delta=18.30\left(\mathrm{C}=\mathrm{C} \mathrm{H}_{3}\right), 28.40$ $\left(\mathrm{C}\left(\underline{\mathrm{C}} \mathrm{H}_{3}\right)_{3}\right), 28.791 \quad 28.84,29.47 \quad\left(\mathrm{OCH}_{2} \underline{\mathrm{CH}_{2}} \mathrm{CH}_{2} \mathrm{~N}\right), 37.51,37.94, \quad 38.14$ $\left(\mathrm{OCH}_{2} \mathrm{CH}_{2} \underline{\underline{C}} \mathrm{H}_{2} \mathrm{~N}\right), \quad 65.77,66.13\left(\mathrm{O}_{\underline{C}} \mathrm{H}_{2} \mathrm{CH}_{2} \mathrm{CH}_{2} \mathrm{~N}\right), 66.67\left(\mathrm{OCH}_{2}\right), 79.31$ $\left(\underline{C}\left(\mathrm{CH}_{3}\right)_{3}\right), 104.41,105.80,106.58,136.42,156.16,156.64,159.77,159.88$ (Ar), $126.04\left(\underline{\mathrm{C}} \mathrm{H}_{2}=\mathrm{C}\right), 136.06\left(\mathrm{CH}_{2}=\underline{C}\right), 167.16,167.60$ (NHCOO) ppm; HRMS-MALDI: $m / z$ (rel-\%): 2525.38 (55) [M - Boc + Na] $]^{+} 2647.39$ (100) [M + $\mathrm{Na}]^{+}, 2663.36(10)[\mathrm{M}+\mathrm{K}]^{+}$; elemental analysis (\%) calcd. for $\mathrm{C}_{135} \mathrm{H}_{198} \mathrm{~N}_{14} \mathrm{O}_{38}$ (2625.12): C 61.77, H 7.60, N 7.47; found: C 61.53, H 7.45, N 7.42.

\section{Poly(3,5-bis(3-(3,5-bis(3-(3,5-bis(3-(tert-butoxycarbonylamino)propoxy) benzamido)propoxy)benzamido)propoxy)benzyl methacrylate) (3c)}

To monomer 3a $(2.91 \mathrm{~g}, 1.11 \mathrm{mmol})$ and AIBN $(2.73 \mathrm{mg}, 0.00166 \mathrm{mmol})$ in a Schlenk Tube was added DMF $(2.36 \mathrm{~g}, 2.5 \mathrm{~mL})$. The mixture was stirred for 30 min. until everything had dissolved. Then the Schlenk tube was weighed 
and vacuum of 2 mbar applied until $574 \mathrm{mg}$. of DMF had been removed. This was typically the case after $2 \mathrm{~h}$ at RT. The obtained highly concentrated solution was still homogenous. Then the Schlenk tube was put into a $65^{\circ} \mathrm{C}$ preheated oil bath. After $24 \mathrm{~h}$ the viscosity had increased to the point that magnetic stirring was not possible anymore, but reaction mixture was continued to be heated for another $12 \mathrm{~h}$. The polymer formed was dissolved in DCM and then purified by column chromatography (silica gel, DCM eluent) which yielded $3 \mathrm{c}$ as colorless foam (1.92 g, 65\%); ${ }^{1} \mathrm{H} \mathrm{NMR}\left(\mathrm{CDCl}_{3} / \mathrm{CD}_{3} \mathrm{OD}\right)$ : $\delta=0.68\left(\mathrm{br}, 2 \mathrm{H}, \mathrm{CH}_{2}\right), 0.88\left(\mathrm{br}, 3 \mathrm{H}, \mathrm{CH}_{3}\right), 1.36\left(\mathrm{br}, 72 \mathrm{H}\right.$; $\left.{ }^{\mathrm{B}} \mathrm{Bu}\right), 1.86(\mathrm{br}, 28 \mathrm{H}$; $\mathrm{OCH}_{2} \mathrm{CH}_{2} \mathrm{CH}_{2}$ ), 3.15 (br, $28 \mathrm{H} ; \mathrm{CH}_{2} \mathrm{NH}$ ), 3.87 (br, $\left.28 \mathrm{H} ; \mathrm{OCH}_{2} \mathrm{CH}_{2} \mathrm{CH}_{2} \mathrm{~N}\right), 4.86$ (br, $2 \mathrm{H}$; OCH${ }_{2} \mathrm{Ph}$ ), $6.46(\mathrm{br}, 6 \mathrm{H} ; \mathrm{Ph}), 6.88(\mathrm{br}, 12 \mathrm{H}, \mathrm{Ph})$ 5.26, 8.26 (br, $12 \mathrm{H}$; $\mathrm{NH})$ ppm; ${ }^{13} \mathrm{C}$ NMR: $\delta=26.97,28.50,29.21,29.61,34.52,37.67,65.96$, $79.26,104.51,106.10,136.45,156.89,160.14,168.27$ ppm; elemental analysis (\%) calcd. for $\left(\mathrm{C}_{135} \mathrm{H}_{198} \mathrm{~N}_{14} \mathrm{O}_{38}\right)_{\mathrm{n}}(2625.12)_{n}$ : C 61.77, H 7.60, N 7.47; found: C 60.86, H 7.45, N 7.27.

\section{Poly(3,5-bis(3-(3,5-bis(3-(3,5-bis(3-aminopropoxy)benzamido)propoxy) benzamido)propoxy)benzyl methacrylate) x $8 \mathrm{CF}_{3} \mathrm{COOH}$ (PG3)}

To $3 \mathrm{c}(1.9 \mathrm{~g}, 0.723 \mathrm{mmol})$, TFA ( $4 \mathrm{~mL}, 6.12 \mathrm{~g}, 53.67 \mathrm{mmol}$ ) was added. After stirring for $24 \mathrm{~h}, 20 \mathrm{~mL}$ of $\mathrm{DCM} /$ methanol was added and reaction mixture was left for another 2d. Evaporation of solvent gave PG3 as brownish solid (1.68 g, $85 \%$ ); ${ }^{1} \mathrm{H}$ NMR ( $\left.\mathrm{CDCl}_{3} / \mathrm{CD}_{3} \mathrm{OD}\right): \delta=0.68\left(\mathrm{br}, 2 \mathrm{H}, \mathrm{CH}_{2}\right), 0.88(\mathrm{br}, 3 \mathrm{H}$, $\left.\mathrm{CH}_{3}\right), 1.97\left(\mathrm{br}, 28 \mathrm{H} ; \mathrm{OCH}_{2} \mathrm{CH}_{2} \mathrm{CH}_{2}\right.$ ), $3.08\left(\mathrm{br}, 28 \mathrm{H} ; \mathrm{CH}_{2} \mathrm{NH}\right), 3.41,4.01(\mathrm{br}$, $28 \mathrm{H} ; \mathrm{OCH}_{2} \mathrm{CH}_{2} \mathrm{CH}_{2} \mathrm{~N}$ ), 4.60 (br, $2 \mathrm{H} ; \mathrm{OCH}_{2} \mathrm{Ph}$ ), 6.48 (br, $6 \mathrm{H}$; Ph), 6.91 (br, $12 \mathrm{H}, \mathrm{Ph}) 8.11\left(\mathrm{br}, 12 \mathrm{H}\right.$; NH) ppm; ${ }^{13} \mathrm{C}$ NMR: $\delta=28.30,30.28,38.48,66.54$, 67.23, 105.79, 107.29, 137.66, 161.14, 161.49, 169.59 ppm; elemental analysis (\%) calcd. for $\left(\mathrm{C}_{111} \mathrm{H}_{142} \mathrm{~N}_{14} \mathrm{O}_{38} \mathrm{~F}_{24}\right)_{n}(2736.38)_{n}$ : C $48.72, \mathrm{H} 5.23 \mathrm{~N}$ 7.17; found: C 46.75, H 5.19 N 6.66. 


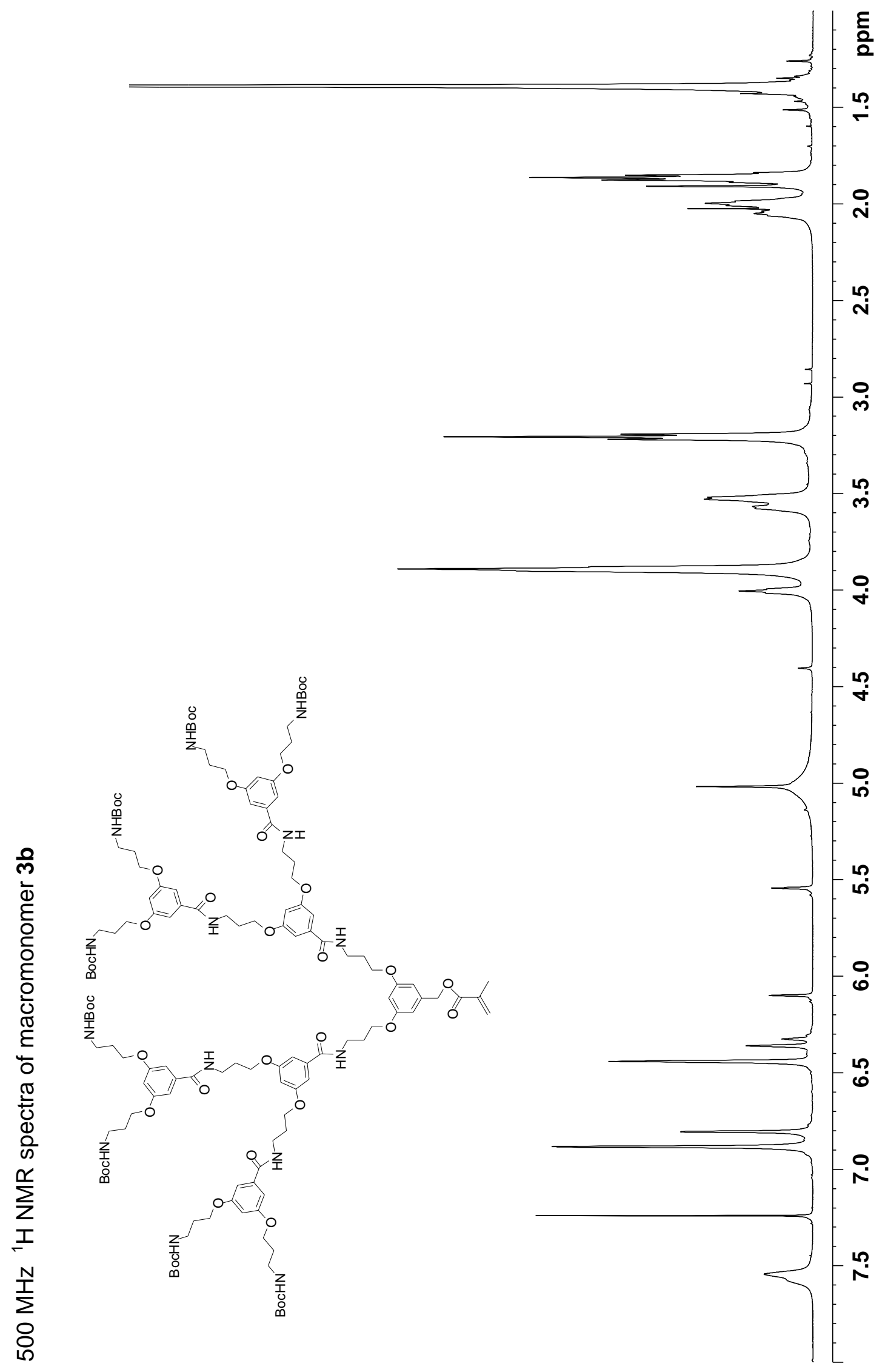

